\title{
Time course of changes in in vitro lipolysis of intra-abdominal fat depots in relation to high-fat diet-induced hepatic steatosis in rats
}

\author{
Pascal Collin ${ }^{1}$, Natalie Chapados ${ }^{1}$, Elise Dufresne ${ }^{1}$, Pierre Corriveau ${ }^{1}$, Pascal Imbeault ${ }^{2}$ \\ and Jean-Marc Lavoie ${ }^{1 *}$ \\ ${ }^{1}$ Département de kinésiologie, Université de Montréal, C.P. 6128, Succ. centre-ville, Montréal, Québec, Canada H3C $3 J 7$ \\ ${ }^{2}$ School of Human Kinetics, University of Ottawa, 125 University Street Ottawa, KIN 6N5, Ontario, Canada
}

(Received 12 September 2005 - Revised 6 January 2006 - Accepted 7 February 2006)

\begin{abstract}
The purpose of the present study was to determine the time course of changes in in vitro lipolysis and in perilipin content (Western blot) in the mesenteric and/or the retroperitoneal fat depots in relation to the development of hepatic steatosis in high-fat diet-fed rats. Female Sprague-Dawley rats were submitted to a high-fat diet (HF diet; $42 \%$ as kJ) or a standard diet (SD diet) for 1, 2, 3 or 8 weeks. Fat accretion in the mesenteric and retroperitoneal tissues was higher $(P<0.01)$ in HF diet-fed than in SD diet-fed rats as soon as 1 week after the beginning of the diet. Liver triacylglycerol concentrations were significantly $(P<0 \cdot 01)$ higher in HF diet-fed than in SD diet-fed rats throughout the experiment, the highest values being reached at week 2 of the diet. Basal and stimulated lipolysis $\left(10^{-4}\right.$ to $10^{-7} \mathrm{M}$-isoproterienol $)$ in the mesenteric and retroperitoneal fat depots was not changed during the first 3 weeks, regardless of the diet. Lipolysis in the mesenteric adipose tissue in the basal and stimulated states was, however, higher $(P<0.01)$ in HF diet-fed than in SD diet-fed rats after 8 weeks of the diets. There were no significant $(P>0.05)$ effects of diet and time on perilipin content of mesenteric tissue. In spite of a rapid fat accretion, the present results do not provide any evidence of a rapid (3 weeks) increase in in vitro lipolysis in intra-abdominal fat depots upon the undertaking of an HF diet at a time where liver lipid infiltration is the most significant.
\end{abstract}

Perilipin: Plasma NEFA: Leptin: Hepatic steatosis: Isoproterenol-induced lipolysis

The pathogenic contribution of obesity to several diseases, namely hypertension and diabetes, is now recognized as a major public health problem worldwide (Must et al. 1999). In recent years, non-alcoholic fatty liver disease has emerged as a new clinical problem among obese patients (Festi et al. 2004). Although non-alcoholic fatty liver disease is a syndrome with a multifactorial aetiology (Chitturi \& Farrell, 2001), obesity is the most common associated factor (James \& Day, 1999). The large majority of obese subjects have evidence of fatty liver (Bellantani et al. 2000). Hepatic fat accumulation has been linked to insulin resistance and the development of metabolic diseases (Marchesini et al. 2001). It, thus, becomes imperative to gain as much information as possible on how the liver becomes infiltrated with lipids.

There is little information on the time course of the development of liver lipid infiltration. Studies using an animal model submitted to diets rich in lipids indicate that the liver may accumulate lipids within a few weeks and even a few days (Gauthier et al. 2004; Samuel et al. 2004). The reason for this rapid liver lipid infiltration, however, is not clear. Increased delivery of NEFA, increased hepatic synthesis of NEFA, decreased NEFA $\beta$-oxidation in the liver and/or decreased synthesis or secretion of VLDL are key factors (Festi et al. 2004). Among these factors the increased NEFA flow to the liver is generally considered as the most important. The increased NEFA flow to the liver may be induced by an increased release of NEFA from adipocytes, excess lipid content in the diet and increased endogenous synthesis of NEFA in the liver (Lewis et al. 2002). Animal as well as human studies have suggested that the visceral adipose tissue may be a major source of increased NEFA flow to the liver (Scheen \& Luyckx, 2002). It is postulated that increased fat accumulation within adipocytes results in an increased visceral lipolysis rate which may in turn favour the delivery of NEFA to the liver. In the present study, we sought to determine the time course of changes in lipolysis in two intraabdominal adipose tissues, using an in vitro approach, and to assess whether these changes may be associated with the time course of changes in liver lipid infiltration observed during the first few weeks of a high-fat diet (HF diet).

In recent years, a new component regulating the activity of hormone-sensitive lipase-mediated lipolysis has been identified. Perilipins are phosphoproteins in adipocytes localized at the surface of the lipid droplet (Greenberg et al. 1991). There is evidence that the access of hormone-sensitive lipase to the lipid droplets is determined by perilipin A, the main isoform of perilipin (Londos et al. 1999; Brasaemle et al. 2000). The second purpose of the present study was to establish the 
time course of the changes in perilipin content in the mesenteric tissue and determine whether these changes may be associated with the time course of changes in in vitro lipolysis in this tissue following the undertaking of an HF diet.

\section{Methods}

Animal care

Female Sprague-Dawley strain rats ( $n$ 80; Charles River, St-Constant, PQ, Canada), weighing 180-200 g (6 weeks of age) upon their arrival were housed individually and had $a d$ libitum access to food and tap water. Their environment was controlled in terms of light ( $12 \mathrm{~h}$ light: $12 \mathrm{~h}$ dark cycle starting at $06 \cdot 00$ hours), humidity and room temperature $\left(20-23^{\circ} \mathrm{C}\right)$. All experiments described in the present report were conducted according to the directives of the Canadian Council on Animal Care.

\section{Diet protocol}

A few days after their arrival, animals were randomly assigned to a standard diet (SD diet) or an HF diet for 1, 2, 3 or 8 weeks (nine rats per group), while a group of rats were immediately killed to provide for baseline values (time $0 ; n 9$ ). The HF diet consisted of $42 \%$ of total energy as lipids (80\% lard, $20 \%$ maize oil), $36 \%$ as carbohydrate and $22 \%$ as protein, and was provided in small pellets from ICN Pharmaceuticals (New York, USA). The SD diet $(12.5 \%$ of total energy as lipids, $63.2 \%$ as carbohydrate and $24.3 \%$ as protein) consisted of usual pellet rat chow (Agribrands Purina Canada, Woodstock, Ontario, Canada). Details of the diets are described elsewhere (Gauthier et al. 2003).

\section{Blood and tissue sampling}

At the end of their respective diet duration, all animals were weighed and killed between 08.30 and 10.30 hours. Food was removed from the animals' cage at least $2 \mathrm{~h}$ before the animals were killed. After complete anaesthesia (sodium pentobarbital, $50 \mathrm{mg} / \mathrm{kg}$, intraperitoneal injection), the abdominal cavity was rapidly opened following the median line of the abdomen. Blood was rapidly $(<45 \mathrm{~s})$ drawn from the abdominal vena cava $(\sim 4 \mathrm{ml})$ into syringes pretreated with EDTA $(15 \%)$. Blood was centrifuged $\left(4000 \mathrm{rpm}\right.$ for $\left.10 \mathrm{~min}, 4^{\circ} \mathrm{C}\right)$ and the plasma kept for NEFA, glucose and leptin determinations. Shortly after, liver was excised, the median lobe freeze-clamped and used for triacylglycerol determinations. The mesenteric and retroperitoneal fat depots were, thereafter, rapidly excised and weighed. Mesenteric fat pad consisted of adipose tissue surrounding the gastro-intestinal tract from the gastro-oesophageal sphincter to the end of the rectum with special care taken in distinguishing and removing pancreatic cells. Retroperitoneal fat pad was taken as that distinct deposit behind each kidney along the lumbar muscles. Approximately $700 \mathrm{mg}$ of the depots were immediately used for determination of lipolysis. The remaining portion of the mesenteric and retroperitoneal depots as well as plasma and liver samples were stored at $-78^{\circ} \mathrm{C}$ until analyses.

\section{Preparation of isolated adipocytes}

Adipocytes were isolated according to the method of Rodbell (1964). Tissue fragments $(700 \mathrm{mg}$ ) from the mesenteric tissue were minced in polyethylene vials containing Krebs-Ringer bicarbonate buffer (KRBA) in the presence of $1 \mathrm{mg} / \mathrm{ml} \mathrm{col-}$ lagenase P (Roche 1213865; Roche Diagnostics, Mannheim, Germany). KRBA also contained fatty acid-free $4 \%$ bovine serum albumin and $0.5 \mathrm{~g} / \mathrm{l}$ glucose. Thereafter, the atmosphere of the flasks was saturated with $95 \% \mathrm{O}_{2}$ and $5 \% \mathrm{CO}_{2}$. Adipocytes were then incubated in a shaking water bath at $37^{\circ} \mathrm{C}$ for $30 \mathrm{~min}$ with a shaking frequency of 100 strokes/min. The digested pieces were filtered through a nylon mesh for separation of fat cells from the stroma. The filtrate was collected in a graduated polyethylene tube to avoid cell breakage. The aqueous phase was aspirated with polyethylene pipettes. The floating adipocytes were then resuspended in $5 \mathrm{ml}$ fresh KRBA. This process was repeated three times to eliminate the remaining collagenase. The adipocytes were stored in the KRBA for 2 min between two washes.

\section{Incubation of adipocytes and measurement of lipolysis}

Basal and stimulated lipolysis were determined in isolated adipocytes $(50 \mu \mathrm{l})$ incubated with a $10 \mu \mathrm{l}$ solution of KRBA and ascorbic acid (basal) and with isoproterenol adjusted to give concentrations of $10^{-7}, 10^{-5}$ and $10^{-4} \mathrm{M}$. Isoproterenol was used because it is a pure $\beta$-agonist and as such it elicits the greatest lipolytic response (Berger \& Barnard, 1999). The incubation was performed at $37^{\circ} \mathrm{C}$ under a $95 \% \mathrm{O}_{2}-5 \%$ $\mathrm{CO}_{2}$ atmosphere with gentle shaking (80 strokes/min) for $2 \mathrm{~h}$. The reaction was stopped by adding $50 \mu \mathrm{l} \mathrm{HCl}(1 \mathrm{M})$, and then $50 \mu \mathrm{l} \mathrm{NaOH}(1 \mathrm{M})$ to neutralize the medium. The vials were vortexed and put on ice for $10 \mathrm{~min}$. After centrifugation for $5 \mathrm{~s}$ at $250 \mathrm{~g}$, the aqueous phase was removed and stored at $-78^{\circ} \mathrm{C}$ until glycerol determination.

Lipolytic activity was measured by glycerol quantification with the use of commercially available kits from Roche Diagnostics. Glycerol quantification was expressed in millions of cells per $\mathrm{h}$ after counting the number of cells in each tested sample.

\section{Cell count}

The number of cells in each individual sample was determined by first diluting $100 \mu \mathrm{l}$ of the isolated adipocyte preparation into $9.9 \mathrm{ml} \mathrm{KRBA}(100 \mu \mathrm{l})$ and then dropping $50 \mu \mathrm{l}$ of this solution in $25 \mu$ l trypan blue $(0.4 \%$ normal saline). The total number of cells in this final suspension was determined with the use of a Nikon-optiphot microscope $(10 \times)$ and quantified with Image-Pro plus version 4.5 software (Media Cybernetics, Silver Spring, MD, USA).

\section{Measurement of perilipin content}

Perilipin content was measured only in the mesenteric tissue. Mesenteric fat depots $(\sim 100 \mathrm{mg})$ were homogenized in EDTA-sucrose buffer and centrifuged at $12000 \mathrm{~g}$ at $4^{\circ} \mathrm{C}$ for $20 \mathrm{~min}$. The infranatant was collected with a blunt-tipped Pasteur pipette and stored at $-80^{\circ} \mathrm{C}$ until perilipin determination. Perilipin A content in adipocytes was determined by Western blotting. 
All samples (25 $\mu$ g proteins) were separated on a $10 \%$ SDSpolyacrylamide gel followed by protein transfer to a polyvinylidene difluoride membrane by electroblotting. The membrane was blocked in $5 \%(\mathrm{w} / \mathrm{v})$ skim milk in TBS (100 mM-Tris, $100 \mathrm{~mm}-\mathrm{NaCl}, \mathrm{pH} 7.5$ ) for $2 \mathrm{~h}$ at room temperature before overnight incubation with a rat perilipin A polyclonal antibody (ABR-Affinity Bio Reagents Golden, CO, USA) with $0.05 \%$ sodium azide. After three washes in TBS-Triton $0.5 \%$, the membrane was incubated for $1 \mathrm{~h}$ with an anti-rabbit IgG peroxidase-conjugated antibody (Jackson Immuno Research Laboratories Inc. West Grove, PA, USA) diluted to $0.8 \mu \mathrm{g} / \mathrm{ml}$ in $0.1 \%$ bovine serum albumin, $0.1 \%$ Tween 20 and $1 \%(\mathrm{w} / \mathrm{v})$ skim milk in TBS. The membrane was washed three times for $10 \mathrm{~min}$ each time in TBS-Triton $0.5 \%$ before a chemifluorescence substrate (enhanced chemiluminescence; Amersham, Baie D'Urfée, $\mathrm{PQ}$, Canada) was applied to the membrane. The resulting signal was detected on enhanced chemiluminescence film (Amersham), scanned with the use of Agfas Arcus II, quantified with Image-Pro plus version 4.5 software and expressed as arbitrary units.

\section{Analytical procedures}

Plasma glucose concentration was determined with the use of a glucose analyser (Yellow Springs Instruments 2300, Yellow Springs, OH, USA). Plasma insulin levels were measured with commercially available kits from MP Biomedicals, LLC (Orangeburg, NY, USA). Plasma NEFA levels were measured with commercially available kits from Roche Diagnostics. Leptin levels were measured with commercially available kits from Linco (St Charles, MO, USA). Liver triacylglycerol concentration was estimated from glycerol released after ethanolic $\mathrm{KOH}$ hydrolysis using a commercial kit (Sigma Diagnostics, St Louis, MO, USA). Although this method does not discriminate between glycerol from phospholipids or triacylglycerol, Frayn \& Maycock (1980) have shown that omitting removal of phospholipids leads to only a $\pm 2 \%$ error in the determination of tissue triacylglycerol.

\section{Statistical analysis}

Values are expressed as means with their standard errors. Statistical analyses were performed by a two-way ANOVA for non-repeated-measures design with diet and time as main effects at times $1,2,3$ and 8 weeks, excluding time-point 0 (since no diet treatment was given at that time). In a second step, the effect of time was analysed separately in HF dietfed and in SD diet-fed rats using a one-way ANOVA for non-repeated measures over all time-points using the same values at time 0 for both groups, with the exception of the lipolysis data in the mesenteric tissue for which the number of observations obtained at time 0 was too small. This second statistical analysis was performed to characterize better the effect of time in each diet including comparison with time 0. Fisher's post-hoc test was used in the event of a significant $(P<0.05) F$ ratio.

\section{Results}

There was no statistical $(P>0.05)$ difference in body weight between SD diet-fed and HF diet-fed rats at any time-points with the exception of time 2 weeks (Table 1). Significantly $(P<0.01)$ higher values of liver triacylglycerol, plasma NEFA and leptin concentrations were found in HF diet-fed compared to SD diet-fed rats as soon as 1 week and throughout the 8-week experimental period (Fig. 1(A-C)). That the HF diet resulted in a rapid and transient liver lipid infiltration is indicated by the significant $(P<0.05)$ increase in liver triacylglycerol observed only after 1 and 2 weeks compared to time 0 . There was no change in liver lipid infiltration in SD diet-fed rats. A significant $(P<0.05)$ increase in plasma NEFA and leptin concentrations over time was found as soon as 2 and 1 weeks, respectively, after introducing the HF diet. In SD diet-fed rats a significant $(P<0.01)$ increase over time was found only after 8 weeks. Plasma glucose concentrations significantly $(P<0.05)$ and similarly increased over time in both diet conditions (Fig. 1(D)). There were no significant effects of diet and time on plasma insulin response with the exception of the values measured at time 2 weeks in the HF diet condition (Table 1).

The HF diet resulted in significantly $(P<0 \cdot 01)$ higher relative weights of the mesenteric and retroperitoneal fat pad at all times compared to SD diet-fed animals (Fig. 2). Compared to time 0 , HF diet-fed and SD diet-fed rats showed significantly

Table 1. Body weight and plasma insulin concentrations at time 0 and in rats fed a standard diet (SD diet) or a highfat diet (HF diet) for 1, 2, 3 and 8 weeks§

\begin{tabular}{|c|c|c|c|c|c|c|c|c|c|c|}
\hline & \multicolumn{10}{|c|}{ Weeks } \\
\hline & \multicolumn{2}{|c|}{0} & \multicolumn{2}{|c|}{1} & \multicolumn{2}{|c|}{2} & \multicolumn{2}{|c|}{3} & \multicolumn{2}{|c|}{8} \\
\hline & Mean & SEM & Mean & SEM & Mean & SEM & Mean & SEM & Mean & SEM \\
\hline \multicolumn{11}{|c|}{ Body weight (g)\| } \\
\hline SD diet & 236 & 3 & $244 \dagger \dagger$ & $2 \cdot 2$ & $265 \dagger \dagger$ & $2 \cdot 3$ & 284†† & 6.4 & $320 \dagger \dagger$ & $14 \cdot 8$ \\
\hline HF diet & - & & $258 \dagger$ & $7 \cdot 3$ & $277^{\star \star} \dagger \dagger$ & 3.7 & 293†† & $8 \cdot 7$ & $352 \dagger \dagger$ & $13 \cdot 2$ \\
\hline \multicolumn{11}{|c|}{ Insulin $(p m o l / l) \|$} \\
\hline SD diet & $69 \cdot 3$ & 14 & $78 \cdot 3$ & 21 & 146 & 22 & 111 & 16 & 111 & 26 \\
\hline HF diet & - & & 156 & 30 & 44††キ & 11 & 128 & 29 & 177 & 51 \\
\hline
\end{tabular}

Mean values were significantly different between SD diet-fed and HF diet-fed rats: ** $P<0.01$

Mean values were significantly different (time 0 ) from baseline values: $\dagger P<0.05 ; \dagger \dagger P<0.01$.

Mean values were significantly different from values at time 1,3 , and 8 weeks: $\ddagger P<0.05$.

$\S$ For details of procedures, see p. 269.

॥ Nine rats per group for body weight and six to nine rats per group for insulin, with the exception of time 0 and 1 week (SD diet), two and three rats, respectively. 

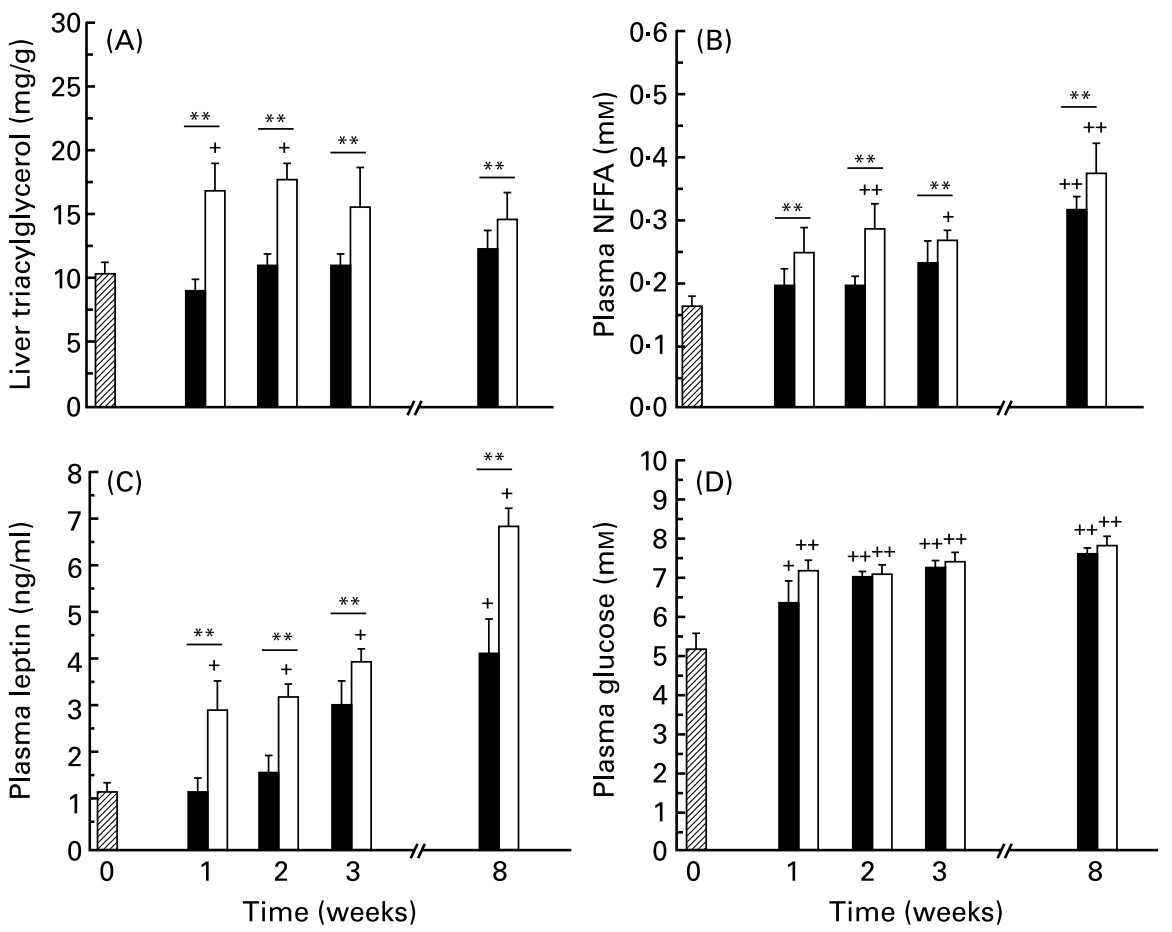

Fig. 1. Liver triacyglycerol (A), plasma NEFA (B), plasma leptin (C) and plasma glucose (D) concentrations at time 0 (baseline values) and in rats fed a standard diet $(\square)$ or a high-fat diet $(\square)$ for 1, 2, 3 and 8 weeks. For details of procedures, see p. 269. Values are means with their standard errors depicted by vertical bars (seven to ten rats per group, with the exception of two and five rats for leptin at times 0 and high-fat diet 1 week, respectively). Mean values were significantly different between standard and high-fat diet-fed rats: ${ }^{\star \star} P<0.01$. Mean values were significantly different from basal values (time 0 ): ${ }^{+} P<0.05$; ${ }^{++} P<0.01$.

$(P<0 \cdot 05)$ higher values in the mesenteric fat pad weights after 2 and 8 weeks, respectively, and after 1 and 8 weeks, respectively, for the retroperitoneal tissue. Inter-group comparisons of lipolysis in the basal condition and following incubation with isoproterenol in the mesenteric and retroperitoneal fat tissues are presented in Figs 3 and 4, respectively. There were no significant differences in basal and stimulated lipolysis between HF diet-fed and SD diet-fed rats at times 1, 2 and 3 weeks in both the mesenteric and the retroperitoneal fat tissues (with two exceptions; Fig. 3(A, C)). Basal and stimulated $\left(10^{-5}, 10^{-4} \mathrm{M}\right)$ lipolytic activities of the mesenteric tissue were, however, significantly $(P<0.05)$ higher in HF diet-fed than in SD diet-fed rats at time 8 weeks (Fig. 3). There were no effects of time (1-8 weeks) on basal and stimulated lipolysis in the mesenteric tissue of rats fed the SD diet. In HF diet-fed rats, however, basal and stimulated lipolytic activities of the mesenteric tissue were significantly $(P<0.05)$ higher at time 8 weeks compared to times 1,2 and 3 weeks (Fig. 3). There were no significant differences in basal and stimulated lipolysis in the retroperitoneal tissue between HF diet-fed and SD diet-fed rats (Fig. 4). However, basal and stimulated $\left(10^{-7} \mathrm{M}\right)$ lipolysis in the retroperitoneal
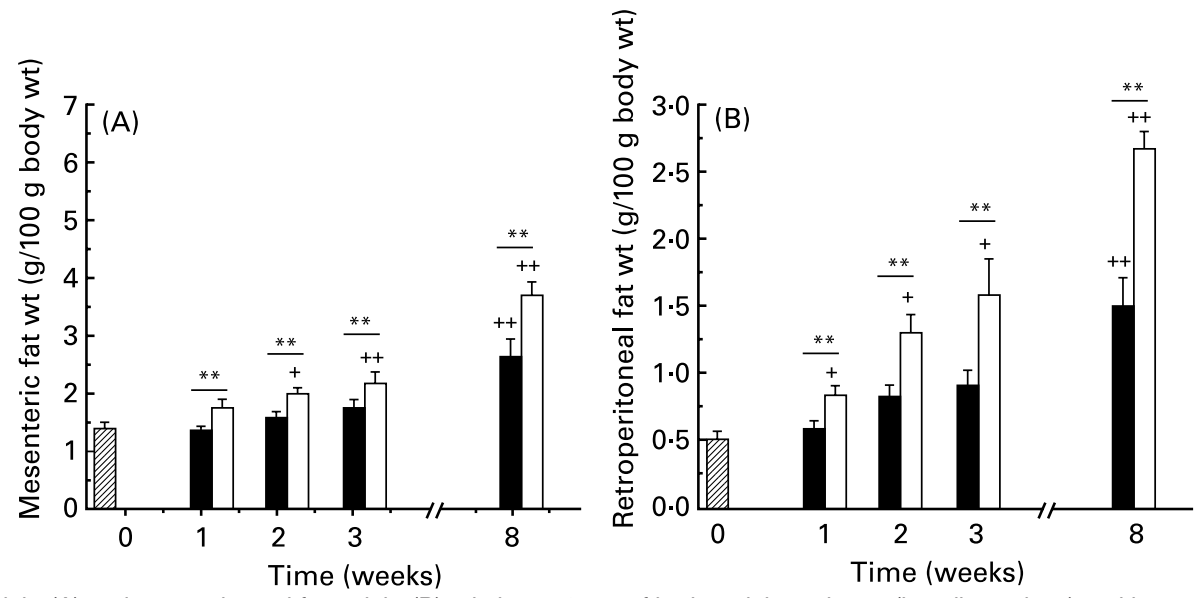

Fig. 2. Mesenteric fat weight (A) and retroperitoneal fat weight (B) relative to $100 \mathrm{~g}$ of body weight at time 0 (baseline values) and in rats fed a standard diet ( $\square$ ) or a high-fat diet $(\square)$ for 1, 2, 3 and 8 weeks. For details of procedures, see p. 269. Values are means with their standard errors depicted by vertical bars (nine rats per group). Mean values were significantly different between standard and high-fat diet-fed rats: ${ }^{* *} P<0 \cdot 01$. Mean values were significantly different from basal values (time 0 ): ${ }^{+} P<0.05 ;{ }^{++} P<0.01$. 

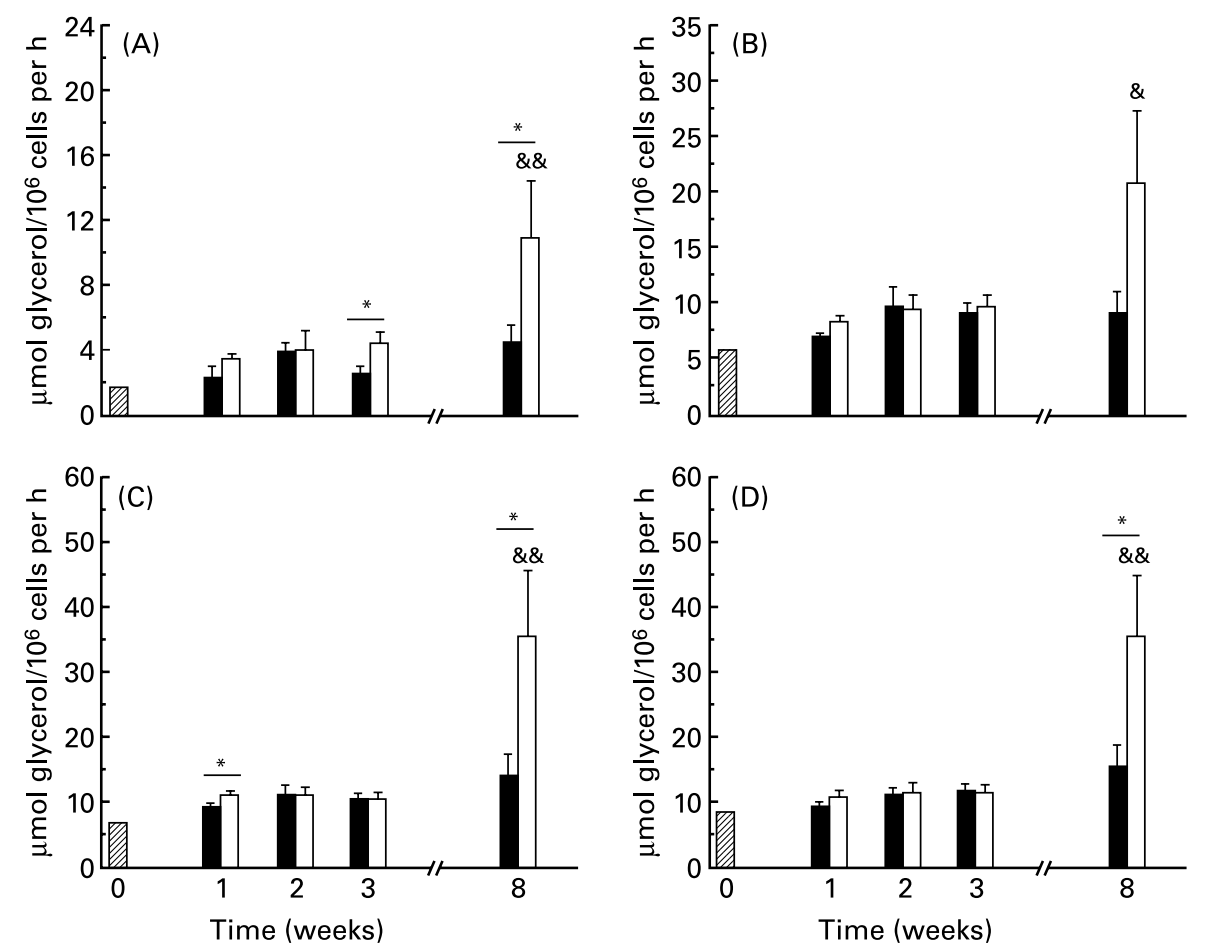

Fig. 3. Adipocyte lipolysis in mesenteric adipose tissue at time 0 (baseline values) and in rats fed a standard diet ( $\square$ ) or a high-fat diet ( $\square$ ) for $1,2,3$ and 8 weeks. For details of procedures, see p. 269. Values are expressed as glycerol production per million cells per $h$ in the basal state $(A)$ and in response to $10^{-7} \mathrm{M}(\mathrm{B})$, $10^{-5} \mathrm{M}(\mathrm{C})$ and $10^{-4} \mathrm{M}(\mathrm{D})$ isoproterenol after $2 \mathrm{~h}$ of incubation. Values are means with their standard errors depicted by vertical bars (seven to nine rats per group, with the exception of time 1 week, five rats). Values at time 0 included only one subject and are given only as a reference value. Time effects were analysed for weeks 1-8 using two-way ANOVA. Mean values were significantly different between standard and high-fat diet-fed rats: ${ }^{\star} P<0.05$. Mean values were significantly different from all other time values: ${ }^{\&} P<0.05$; ${ }^{\& \&} P<0.01$.
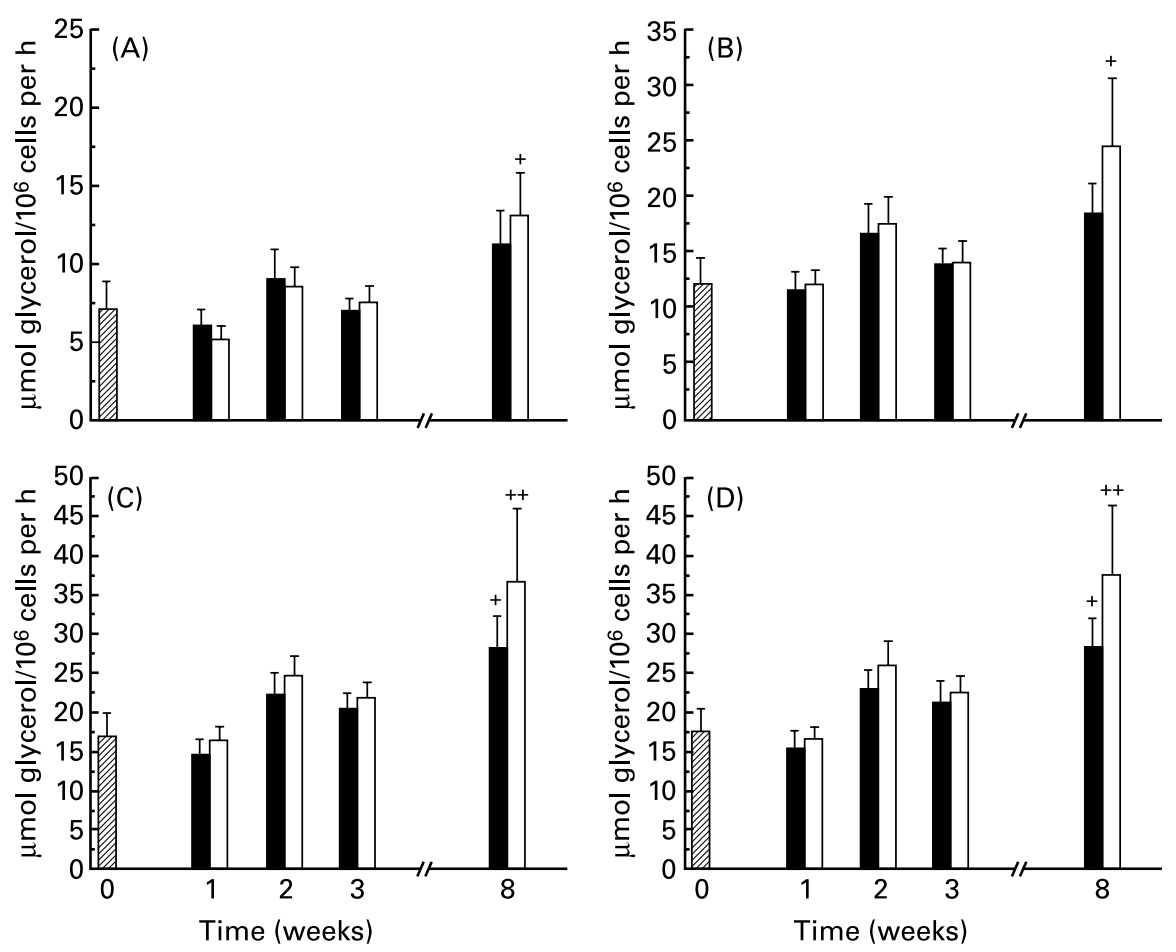

Fig. 4. Adipocyte lipolysis in retroperitoneal adipose tissue at time 0 (baseline values) and in rats fed a standard diet ( $\square$ ) or a high-fat diet ( $\square$ ) for $1,2,3$ and 8 weeks. For details of procedures, see p. 269. Values are expressed as glycerol production per million cells per $\mathrm{h}$ in the basal state (A) and in response to $10^{-7} \mathrm{M}$ $(B), 10^{-5} \mathrm{M}(\mathrm{C})$ and $10^{-4} \mathrm{M}(\mathrm{D})$ isoproterenol after $2 \mathrm{~h}$ of incubation. Values are means with their standard errors depicted by vertical bars (seven to nine rats per group). Mean values were significantly different from basal values (time 0 ): ${ }^{+} P<0.05 ;{ }^{++} P<0.01$. 
tissue was significantly $(P<0 \cdot 05)$ higher after 8 weeks of high-fat feeding compared to time 0 weeks (Fig. 4).

There were no significant $(P>0.05)$ effects of diet and time on perilipin content of the mesenteric tissue expressed in arbitrary units (Fig. 5(A)). When the perilipin content was expressed taking into account the weight of the mesenteric fat pad, the perilipin to fat ratio was significantly $(P<0 \cdot 01)$ lower in HF diet-fed than in SD diet-fed rats at all timepoints (Fig. 5(B)). There was no effect of time on the perilipin to fat ratio in SD diet-fed rats. However, the perilipin to fat ratio was significantly $(P<0 \cdot 01)$ lower after 8 weeks of high-fat feeding compared to time 0 .

\section{Discussion}

Time course of changes in intra-abdominal tissue lipolysis

The first finding of the present study is that in vitro lipolysis in the mesenteric and the retroperitoneal tissue was not, on the whole, increased in the first 3 weeks of an 8-week HF diet protocol in rats. This response was observed in the basal as well as in the isoproterenol-stimulated state. On the other hand, basal as well as stimulated lipolysis in the mesenteric and, to a lesser extent, in the retroperitoneal tissues was highly increased after 8 weeks of high-fat feeding. It is generally assumed that fat accumulation in the adipocyte leads to an increased release of fatty acids through an increased lipolytic activity (Large \& Arner, 1998). How much accumulation of fat is needed to generate an increased lipolytic activity, however, is unknown. Fat accumulation in the mesenteric and the retroperitoneal tissue, as well as plasma leptin levels, were significantly increased as soon as 1 week after the undertaking of the HF diet. It is not until the mesenteric and the retroperitoneal fat reached an accumulation between 2.5 and 3 times the baseline values that a significant increase in lipolytic activity was observable. These data indicate that a substantial amount of lipids must accumulate inside the adipocytes before the lipolytic activity of these cells is increased. On the whole, the present results indicate that, within the limits of the in vitro situation, lipolysis in the mesenteric and retroperitoneal tissues is not rapidly changed upon the undertaking of an HF diet in rats.

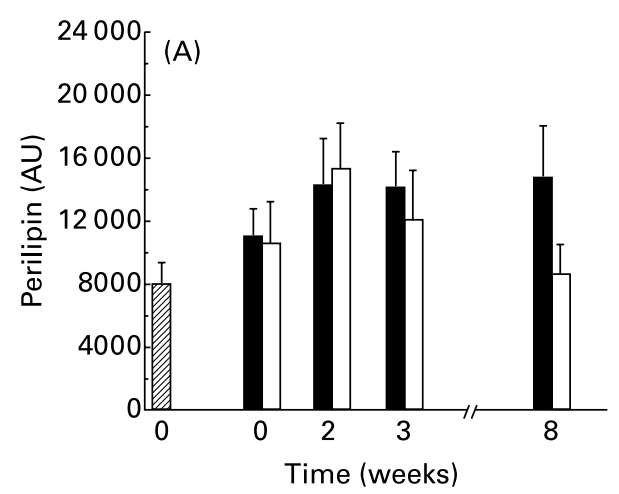

\section{Lipolysis and liver lipid infiltration}

It has now become notorious that chronic obesity is associated with a state of hepatic steatosis (Festi et al. 2004). Recent data have provided evidence that hepatic fat may accumulate rapidly upon the undertaking of a high fat diet (Gauthier et al. 2005). The significance of this infiltration is not negligible since rapid hepatic fat accumulation, even as a matter of days, has been reported to result in hepatic insulin resistance (Kraegen et al. 1991; Samuel et al. 2004). The HF diet used in the present experiment resulted in a large increase in hepatic fat accumulation as soon as 1 week after introducing the HF diet. Indeed, the difference in hepatic triacylglycerols between HF diet-fed and SD diet-fed rats was more important in the first few weeks than it was after 8 weeks. Increased hepatic fat accumulation is strongly associated with increased circulatory NEFA levels and increased adipocyte fat deposition (Gauthier et al. 2003; Sniderman et al. 1998). Accordingly, plasma NEFA levels and fat accumulation in the mesenteric and retroperitoneal tissues were rapidly increased after the undertaking of the HF diet. However, the origin of the additional circulating NEFA, under this circumstance, is still nebulous. Interestingly, basal as well as stimulated lipolysis of the adipocytes of the mesenteric and retroperitoneal tissues were not, as a whole, increased in the first 2 weeks of the $\mathrm{HF}$ diet, at a time where the increase in hepatic fat accumulation was the most significant. The association between the present in vitro lipolytic response in the intra-abdominal fat depots and the hepatic fat accumulation following the HF diet is limited by the in vitro versus the in vivo aspects of these measurements, respectively. Notwithstanding this limitation, the present results strongly suggest that an increased lipolytic activity was not at the origin of the rapid increase in plasma NEFA and hepatic fat accretion upon the undertaking of a HF diet. On the other hand, basal lipolysis in the fat depots was highly increased after 8 weeks of high-fat feeding, and very likely constitutes a source of circulating NEFA that may result in liver lipid infiltration on a longer-term basis. The important increase in in vitro lipolysis in the mesenteric and, to a lesser extent, the retroperitoneal tissue after 8 weeks of high-fat feeding supports the contention of an increased release of NEFA from adipose tissue in patients with excess

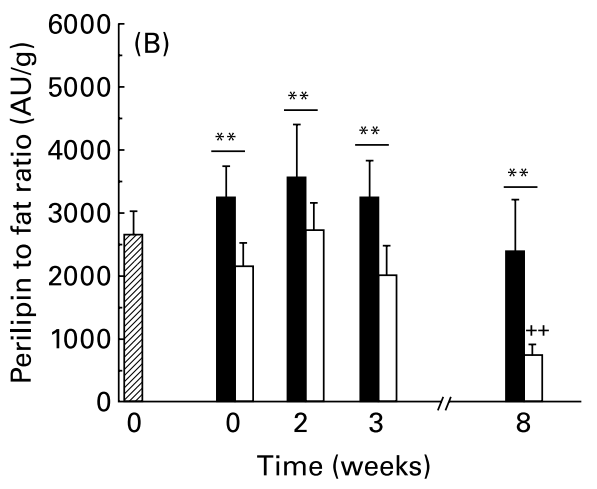

Fig. 5. Perilipin content (A) and perilipin to fat ratio (B) measured in mesenteric tissue from rats fed a standard diet ( $\square$ ) or a high-fat diet ( $\square$ ) for 1,2 , 3 and 8 weeks. For details of procedures, see p. 269. Values are means with their standard errors depicted by vertical bars (eight or nine rats per group, with the exception of time 0 , six rats). Mean values were significantly different between standard and high-fat diet-fed rats: ${ }^{* *} P<0.01$. Mean values were significantly different from basal values (time 0 ): ${ }^{++} P<0.01$. Perilipin was measured by Western blotting and expressed in arbitrary units (AU). Perilipin to fat ratio was obtained by dividing the perilipin content by the absolute weight $(\mathrm{g})$ of the mesenteric depot. 
weight (Frayn 2000; Mottagui-Tabar et al. 2003). On the whole, however, data of the present investigation indicate that even though fat accretion in adipocytes and plasma NEFA levels rapidly increase, the rapid gain in hepatic fat accretion upon the undertaking of an $\mathrm{HF}$ diet regimen in rats is not paralleled by an increased in vitro lipolysis activity.

The observation that in vitro lipolysis in the intra-abdominal fat depots is not rapidly ( 3 weeks) increased upon the undertaking of a HF diet raises the question as to where the lipids stored in the liver come from. Although they cannot be ruled out, it is unlikely that intra-hepatic factors such as increased hepatic synthesis of NEFA, decreased NEFA $\beta$-oxidation in the liver, and/or decreased synthesis or secretion of VLDL play an important role in developing liver lipid infiltration under the present HF diet regimen. In the absence of a significant rapid increase in in vitro lipolysis in intra-abdominal fat depots, it is postulated that the present increase in liver lipid infiltration is related to the increased lipid content of the diet through the uptake of intestinally derived chylomicron remnants (Havel \& Hamilton, 2004) and through the spillover of the activity of the lipoprotein lipase and the amount of circulating NEFA generated by this activity (Miles et al. 2004). The observation that plasma NEFA levels were indeed increased after only 1 week of highfat feeding points toward the direction of an elevated rate of escape of NEFA from esterification in adipose tissue. In this respect, Riemens et al. (2000) recently suggested that the elevated NEFA transport rate during fasting in patients with type 2 diabetes is an elevated rate of escape of NEFA from tissue uptake in adipose tissue.

\section{Perilipin and basal lipolysis}

We did not find any changes in perilipin content in absolute values with the HF diet over time (Fig. 5(A)). As a consequence, when perilipin content values were reported per unit of the mesenteric fat depot, thus taking into account the amount of fat accumulated by the cells, the relative values were significantly lower in HF diet-fed than in SD diet-fed rats throughout the 8 -week period. In spite of this, and in spite of a significant accumulation of fat in the adipocytes, basal lipolysis measured in vitro was not increased in the first few weeks of HF diet-fed rats. This may suggest that the initial perilipin content was sufficient to incorporate newly formed triacylglycerols and to protect them from being hydrolysed by hormone-sensitive lipase. It is not until 8 weeks of high-fat feeding that the lowered ratio of perili$\mathrm{pin} / \mathrm{fat}$ accretion is associated with an increase in lipolysis. This is compatible with a more advanced state of obesity (Wang et al. 2003). Overall, it appears that perilipin content in the current study was not increased so as to keep up with the increased fat accretion in adipocyte and this may be associated with the increased basal lipolysis observed in the mesenteric tissue after 8 weeks of high-fat feeding.

\section{Activated lipolysis and perilipin}

Stimulated lipolysis was not altered by the HF diet in the first 3 weeks, which is in line with what was observed in the basal state. After 8 weeks, however, stimulated lipolysis was higher in HF diet-fed than in SD diet-fed rats. These results are perfectly in line with those reported by Berger \& Barnard (1999) in female Fischer 344 rats fed a HF diet for 2 months. These results are not, however, readily reconcilable with what is known about perilipin action under stimulated conditions. The property of perilipin to suppress lipolysis, presumably by blocking access of lipases to the triacylglycerol core lipids with the droplets, is especially evident under conditions where protein kinase A is not activated (Londos et al. 2005). It seems that perilipin is essential for the translocation of hormone-sensitive lipase during lipolytic activation (Sztalryd et al. 2003). The present increase in activated lipolysis in absolute values in rats fed the HF diet for 8 weeks is, rather, associated with a decrease, not an increase, in perilipin content. One possible explanation might be that perilipin content in the present study was not measured specifically in the phosphorylated form. It must also be kept in mind that other factors in addition to hormone-sensitive lipase and perilipin may also contribute to the lipolytic response (Sztalryd et al. 2003).

One limitation of the data of the present study is the fact that lipolysis was measured in an in vitro condition in an animal model. At the present time, intra-abdominal adipose tissue is hardly accessible for studies in man. In vivo studies in animals have in many instances (i.e. effects of training, intra-abdominal compared with subcutaneaous adipose tissue) confirmed what has been reported using in vitro measurements of lipolysis (Enevoldsen et al. 2000). The present lipolytic data are also limited to $\beta$-adrenergic stimulation since lipolysis is also regulated by other pathways (i.e. insulin and $\alpha 2$ adrenoreceptor-mediated anti-lipolytic activity).

In summary, results of the present study show that basal and stimulated in vitro lipolysis in intra-abdominal fat depots are not increased in the first 3 weeks of an 8-week HF diet regimen in rats at a time where liver lipid infiltration is the most significant. It is postulated that short-term liver lipid infiltration is more related to the lipid content of the diet than from increased lipolytic activity in the intra-abdominal fat depots. Although fat accretion in adipocytes was rapidly (weeks 1-3) increased by the high-fat feeding, the absence of a concomitant increase in lipolysis (weeks 1-3) is not explained by an increase in cytosolic perilipin content.

\section{Acknowledgements}

This work was supported by grants from the Natural Sciences and Engineering Research Council of Canada (J. M. L.) and from the Canadian Institutes of Health Research (J. M. L. and P. I.).

\section{References}

Bellantani S, Saccoccio G, Masutti F, Croce LS, Brandi G, Sasso F, Cristanini G \& Tiribelli C (2000) Prevalence and risk factors for hepatic steatosis in Northern Italy. Ann Intern Med 132, 112-117.

Berger JJ \& Barnard RJ (1999) Effect of diet on fat cell size and hormone-sensitive lipase activity. J Appl Physiol 87, 227-232.

Brasaemle DL, Rubin B, Harten IA, Gruia-Gray J, Kimmel AR \& Londos C (2000) Perilipin A increases triacylglycerol storage by decreasing the rate of triacyglycerol hydrolysis. $J$ Biol Chem 275, 38486-38493. 
Chitturi S \& Farrell GC (2001) Etiopathogenesis of non-alcoholic steatohepatitis. Semin Liver Dis 21, 27-41.

Enevoldsen LH, Stallknecht B, Fluckey JD \& Galbo C (2000) Effect of exercise training on in vivo lipolysis in intra-abdominal adipose tissue in rats. Am J Physiol Endocrinol Metab 279, E585-E592.

Festi D, Colecchia A, Sacco T, Bondi M, Roda E \& Marchesini G (2004) Hepatic steatosis in obese patients: clinical aspects and prognostic significance. Obes Rev 5, 27-42.

Frayn KN (2000) Visceral fat and insulin resistance-causative or correlative? Br J Nutr 83, S71-S77.

Frayn KN \& Maycock PF (1980) Skeletal muscle triacylglycerol in the rat: methods for sampling and measurement and studies of biological variability. J Lipid Res 21, 139-144.

Gauthier M-S, Couturier K, Charbonneau A \& Lavoie J-M (2004) Effects of introducing physical training in the course of a 16-week high-fat diet regimen on hepatic steatosis, adipose tissue fat accumulation, and plasma lipid profile. Int J Obes 28, 1064-1071.

Gauthier M-S, Couturier K, Latour JG \& Lavoie J-M (2003) Concurrent exercise prevents high-fat-diet-induced macrovesicular hepatic steatosis. J Appl Physiol 94, 2127-2134.

Gauthier M-S, Favier R \& Lavoie J-M (2006) Time course of the development of non-alcoholic hepatic steatosis in response to high-fat diet-induced obesity in rats. Br J Nutr 95, 273-281.

Greenberg AS, Egan JJ, Wek SA, Garty NB, Blanchette-Mackie EJ \& Londos C (1991) Perilipin, a major hormonally regulated adipocyte specific phosphoprotein associated with the periphery of lipid storage droplets. J Biol Chem 266, 11341-11346.

Havel RJ \& Hamilton RL (2004) Hepatic catabolism of remnant lipoproteins: where the action is. Arterioscler Thromb Vasc Biol 24, $213-215$.

James O \& Day C (1999) Non-alcoholic steatohepatitis. Another disease of affluence. Lancet 353, 1634-1636.

Kraegen EW, Clark PW, Jenkins AB, Daley EA, Chisholm DJ \& Storlien LH (1991) Development of muscle insulin resistance after liver insulin resistance in high-fat-fed rats. Diabetes 40, 1397-1403.

Large V \& Arner P (1998) Regulation of lypolysis in humans. Pathophysiological modulation in obesity, diabetes, and hyperlipidaemia. Diabetes Metab 24, 409-418.

Lewis GF, Carpentier A, Adeli K \& Giacca A (2002) Disordered fat storage and mobilization in the pathogenesis of insulin resistance and type 2 diabetes. Endocrine Rev 23, 201-229.

Londos C, Brasaemle DJ, Schultz CJ, Segrest JP \& Kimmel AR (1999) Perilipins, ADRP, and other proteins that associate with intracellular neutral lipid droplets in animal cells. Semin Cell Dev Biol 10, 51-58.

Londos C, Sztalryd C, Tansey JT \& Kimmel AR (2005) Role of PAT proteins in lipid metabolism. Biochimie 87, 45-49.

Marchesini G, Brizi M, Bianchi G, Tomassetti S, Bugianesi E, Lenzi M, McCullogh AJ, Natale S, Forlani G \& Melchionda N (2001) Nonalcoholic fatty liver disease. A feature of the metabolic syndrome. Diabetes 50, 1844-1850.

Miles JM, Park YS, Walewicz D, Russell-Lopez C, Windsor S, Isley WL, Coppack SW \& Harris WS (2004) Systemic and forearm triglyceride metabolism: fate of lipoprotein lipase-generated glycerol and free fatty acids. Diabetes 53, 521-527.

Mottagui-Tabar S, Ryden M, Lofgren P, Faulds G, Hoffstedt J, Brookes AJ, Andersson I \& Arner P (2003) Evidence for an important role of perilipin in the regulation of human adipocyte lipolysis. Diabetologia 46, 789-797.

Must A, Spadano J, Coakley EH, Field AE, Colditz G \& Dietz WH (1999) The disease burden associated with overweight and obesity. JAMA 282, 1523-1529.

Riemens SC, Sluiter WJ \& Dullaart RP (2000) Enhanced escape of non-esterified fatty acids from tissue uptake: its role in impaired insulin-induced lowering of total rate of appearance in obesity and type 2 diabetes mellitus. Diabetologia 43, 416-426.

Rodbell M (1964) Metabolism of isolated fat cells I. Effects of hormones on glucose metabolism and lipolysis. J Biol Chem 239, $375-380$.

Samuel VT, Liu Z-X, Qu X, Elder BD, Bilz S, Belfroy D, Romanelli AJ \& Shulman GI (2004) Mechanism of hepatic insulin resistance in non-alcoholic fatty liver disease. J Biol Chem 279, $32345-32353$.

Scheen AJ \& Luyckx FH (2002) Obesity and liver disease. Best Pract Res Clin Endocrinol Met 16, 703-716.

Sniderman AD, Cianflone K, Arner P, Summers LKM \& Frayn KN (1998) The adipocyte, fatty acid trapping, and atherogenesis. Arterioscler Thromb Vasc Biol 18, 147-151.

Sztalryd C, Xu G, Dorward H, Tansey JT, Contreras JA, Kimmel AR \& Londos C (2003) Perilipin A is essential for the translocation of hormone-sensitive lipase during lipolytic activation. J Cell Biol 161, 1011-1012.

Wang Y, Sullivan S, Trujillo M, Lee M-J, Schneider S, Brolin RE, Kang YH, Werber Y, Greenberg AS \& Fried SK (2003) Perilipin expression in human adipose tissues: effects of severe obesity, gender, and depot. Obes Res 11, 930-936. 